\title{
A Product Form Approximation for Arbitrary Discrete Time Networks of Shared Buffer Queues*
}

\author{
Demetres D. Kouvatsos and Jeffrey Wilkinson \\ Computer Systems Modelling Research Group, University of Bradford, \\ Bradford, BD7 1DP, West Yorkshire, England.
}

\begin{abstract}
A product form approximation, based on the principle of maximum entropy (ME), is characterised for arbitrary open discrete time queueing network models (QNMs) of shared buffer ATM switches under the departures first (DF) buffer management policy. Traffic entering and flowing in the network is assumed to be bursty and is modelled by a Compound Bernoulli Process (CBP) with geometrically distributed bulk sizes. Entropy maximisation implies decomposition of the network into individual shared buffer switches which are analysed to obtain cell loss probabilities and mean delays. The ME queue length distribution of a single shared buffer queue under DF policy, together with closed form expressions for the first two moments of the effective flow, play the role of building blocks in the solution process. Typical numerical results are included to demonstrate the utility and computational efficiency of the ME procedure. Comments on current work, involving discrete time finite capacity queues with space priority and correlated traffic, are included.
\end{abstract}

Keywords: discrete time queue, queueing network model (QNM), maximum entropy (ME) principle, compound Bernoulli process (CBP), asynchronous transfer mode (ATM) networks, shared buffer ATM switch.

\section{Introduction}

In fast packet switching architectures of Broadband Integrated Service Digital Networks (B-ISDNs) (c.f. Tobagi [1]) messages are decomposed into fixed length blocks which are individually transmitted through the network. As a consequence, traffic in such networks is essentially discrete and basic operational parameters are known via measurements obtained at discrete points of time. Thus, discrete time queueing network models (QNMs) are natural and realistic tools for representing B-ISDNs and optimising their performance. In this context, arrivals and departures of cells are allowed to occur at the boundary epochs of slots while, during a slot, no cells enter or leave the system.

\footnotetext{
${ }^{*}$ Supported by the Engineering and Physical Sciences Research Council (EPSRC), UK, under grant GR/H/18609 and by iT the Post Office.
} 
Asynchronous Transfer Mode (ATM) switch architectures, the preferred solution for BISDN, have $\mathrm{R}$ input and $\mathrm{R}$ output interconnected ports. Of particular importance are shared buffer ATM switches, incorporating a single memory of fixed size which is shared by all output ports [2]. A finite shared buffer stores incoming cells when the output ports are busy. Cells destined for the same output port can be linked together by an address chain or their addresses can be stored in a FCFS buffer associated with a particular output port. A cell will be lost if, on arrival, it finds either the shared buffer full or the address buffer full. An example of such a switch architecture is the Prélude architecture proposed by CNET [2]. For other examples see $[3,4]$.

Performance approximations for queueing models of shared buffer ATM switch architectures have been suggested in the literature [3-9]. However, there are difficulties and open issues associated with the study of these models, especially in the discrete time domain, due to the simultaneous occurrence of events, including bulk arrivals and departures, at the boundary epochs of slots. Consequently, these models are not analytically tractable except in some trivial or restricted cases. Usually it is necessary to resort to either simulation or numerical methods: simulation is time consuming and cannot easily yield the great precision needed for some rare events, such as cell loss, whilst numerical methods are severely limited in scope - even at the switch level - as system size increases. Thus, there is a great need to consider alternative methodologies leading to both credible and cost effective approximations for performance evaluation of ATM switches and networks.

The principle of Maximum Entropy (ME), a probability inference method [10], has been applied successfully, in conjunction with queueing theoretic mean value constraints, to approximate analysis of both continuous time and discrete time QNMs of arbitrary configurations of single general queues with finite or infinite capacity [11-18]. In particular, the principle has been utilised in the study of general multibuffered and shared buffer queues and closed form expressions have been obtained for queue length distribution, cell loss probability and mean delay [15-17]. In the afore mentioned studies the arrival process at each queue has been assumed to be bursty and was modelled by a compound Bernoulli process (CBP) with geometrically distributed bulk sizes [18]. In this context, the burstiness of the arrival process is captured by the squared coefficient of variation of the arrival process and, subsequently, the size of the incoming bulk. This particular CBP implies a generalised geometric (GGeo) interarrival time distribution, of which the pseudo-memoryless properties facilitate analysis of complex discrete time queues and networks [16,17].

In this paper, a new product form approximation is characterised, based upon the principle of Maximum Entropy (ME), for arbitrary open queueing networks of FCFS (first come, first served) shared buffer queues under the departures first (DF) buffer management policy. As in earlier studies, the traffic in the network is assumed to be bursty and it is represented by a CBP with geometrically distributed bulk sizes. Entropy maximisation implies a decomposition of the network into individual shared buffer queues of GGeo type. These queues are solved in isolation and, together with GGeo type formulae for the first two moments of effective flow [18], play the rôle of effective building blocks in the process of solving the complete network. The credibility of the ME solution of a single shared buffer GGeo type queue and that of GGeo type flow formulae has been verified by extensive simulation and numerical results have been reported in [16] and [17], respectively. Thus, the main objective of this paper is creation of a new and cost effective algorithm for 
computational implementation of the ME product form approximation for arbitrary networks of shared buffer queues.

The ME formalism is introduced in Section 2. The GGeo distribution model is described in Section 3. An outline of the ME solution for a single shared buffer queue is given in Section 4. The product form approximation for an arbitrary network of shared buffer queues together with formulae for the first two moments of effective flow within the network are presented in Section 5. The ME algorithm for a network of shared buffer queues is determined in Section 6. Finally, numerical results and concluding remarks follow in Sections 7 and 8, respectively.

\section{$\underline{\text { Remarks }}$}

Arrivals first (AF) and departures first (DF) buffer management policies for discrete time queues stipulate how a buffer is filled or emptied in the case of simultaneous arrivals and departures at a boundary epoch of a slot [19]. In such cases, according to AF policy, arrivals take precedence over departures while, under DF policy, the opposite effect is observed (see figure 1). Such buffer management policies may play a significant rôle in determination of blocking probabilities in discrete time finite capacity queues $[19,20]$.
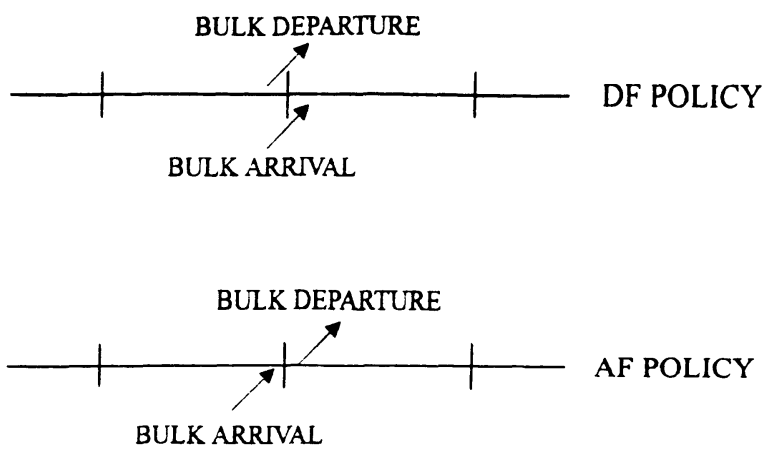

Figure 1. Effects of AF and DF buffer management policies at slot boundary epoch.

\section{Maximum Entropy Formalism}

Consider a system $Q$ which has a set $S$ of possible discrete states $\left\{S_{0}, S_{1}, S_{2}, \ldots\right\}$ which may be finite or countably infinite and state $S_{n}, n=0,1,2, \ldots$ may be specified arbitrarily. Suppose that the available information about $Q$ places a number of constraints on $p\left(S_{n}\right)$, the probability distribution that the system $\mathrm{Q}$ is in state $\mathrm{S}_{n}$. Without loss of generality, it is assumed that these constraints take the form of mean values of suitable functions $\left\{f_{1}\left(S_{n}\right)\right.$, $\left.\mathrm{f}_{2}\left(\mathrm{~S}_{\mathrm{n}}\right), \ldots, \mathrm{f}_{\mathrm{m}}\left(\mathrm{S}_{\mathrm{n}}\right)\right\}$, where $\mathrm{m}$ is less than the number of possible states. The principle of maximum entropy [10] states that, of all distributions which satisfy the constraints, the minimally biased distribution is the one which maximises the system's entropy function

$\mathrm{H}(p)=-\sum_{S_{n} \in S} p\left(S_{n}\right) \ln p\left(S_{n}\right)$

subject to the constraints 
$\sum_{S_{n} \in S} p\left(S_{n}\right)=1$

$\sum_{S_{n} \in S} \mathrm{f}_{k}\left(S_{n}\right) p\left(S_{n}\right)=\left\langle\mathrm{f}_{k}\right\rangle, \quad k=1,2, \ldots, m$

where $\left\{\left\langle\mathrm{f}_{k}\right\rangle\right\}$ are the prescribed mean values defined on the set of functions $\left\{\mathrm{f}_{k}\left(S_{n}\right)\right\}$, $k=1,2, \ldots, m$. The maximisation of (2.1), subject to the constraints (2.2) and (2.3), can be carried out using Lagrange's method of undermined multipliers and leads to the solution

$p\left(S_{n}\right)=\frac{1}{Z} \exp \left(-\sum_{k=1}^{m} \beta_{k} \mathrm{f}_{k}\left(S_{n}\right)\right)$

where $\left\{\beta_{k}\right\}, k=1,2, \ldots, m$, are the Lagrangian multipliers determined from the set of constraints (2.3) and $Z$, known in statistical physics as the "partition function", is given by

$Z=\exp \left(\beta_{0}\right)=\sum_{S_{n} \in S} \exp \left(-\sum_{k=1}^{m} \beta_{k} \mathrm{f}_{k}\left(S_{n}\right)\right)$

where $\left\{\beta_{0}\right\}$ is the Lagrangian multiplier determined by the normalisation constraint (2.2).

Jaynes [21] has shown that, if the prior information includes all constraints actually operative during a random experiment, the distribution predicted by entropy maximisation can be realised in overwhelmingly more ways than by any other distribution. The principle of maximum entropy has also been shown, by Shore and Johnson [22], to provide a "uniquely correct self-consistent method of inference" for estimating probability distributions based on the available information.

Maximum entropy formalism can be used in the performance analysis of queueing systems because expected values of various distributions of interest are usually known in terms of moments of the interarrival and service time distributions. Earlier applications of entropy maximisation for approximate analysis of some continuous time queueing systems and networks can be seen in [11-15].

\section{The GGeo-Type Distribution}

Consider a discrete time random variable (rv) $W$ representing the interarrival time or the service time of a stable single server queue. Let $\mathrm{E}[W]=1 / v$ be the mean and $\mathrm{C}_{W}^{2}$ be the SCV of $W$. The GGeo discrete time distribution [18] is defined by

$\operatorname{Pr}[W=n]= \begin{cases}1-\tau & n=0 \\ \tau \sigma(1-\sigma)^{n-1} & n \geq 1\end{cases}$

where

$\tau=2 /\left(C_{W}^{2}+1+v\right), \quad 0<\tau \leq 1$,

$\sigma=\tau \nu, \quad 0<\sigma<1$,

and $|1-v| \leq C_{W}^{2}$ (see Figure 2). 


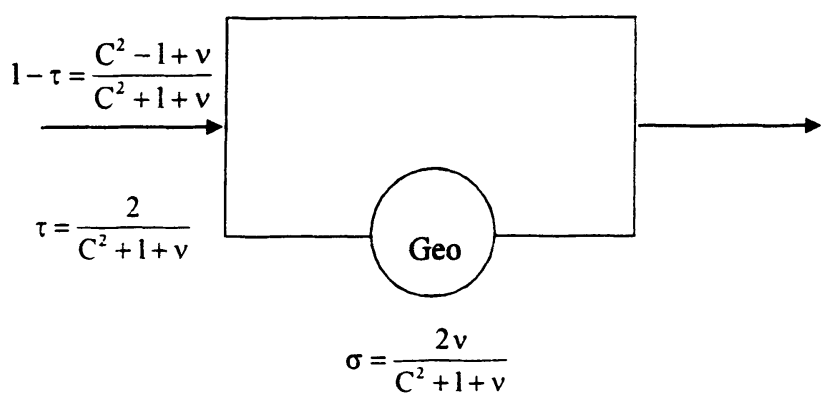

Figure 2. The GGeo distribution with parameters $\tau$ and $\sigma(0<\tau, \sigma \leq 1)$.

Since rv $W$ can realise a value of zero, it is implied that the GGeo distribution accommodates bulk arrivals or departures. In essence, the GGeo distribution corresponds to a CBP with rate $\sigma$, whilst the number of events (i.e. arrivals or departures) in a slot (i.e. the bulk size) is distributed geometrically with parameter $\tau$. Thus, the GGeo pattern comprises a sequence of bulks with independent and identically distributed non-negative integer valued rv's $\left\{W_{k}\right\}$, where $W_{k}, \mathrm{k}=1,2, \ldots$ is the number of events occurring at the $k^{\text {th }}$ slot, with a fixed probability distribution given by

$\operatorname{Pr}\left[W_{k}=\ell\right]= \begin{cases}1-\sigma & \ell=0 \\ \sigma \tau(1-\tau)^{\ell-1} & \ell \geq 1\end{cases}$

It clearly follows that the mean event (arrival or departure) rate is equal to $v=\sigma / \tau$ events per slot. The $\operatorname{GGeo}(\tau, \sigma)$ distribution is versatile, possessing some interesting properties, for example:

i) the remaining interevent time of a $\operatorname{GGeo}(\tau, \sigma)$ distribution is geometric with parameter $\sigma$ (the "pseudo memoryless" property);

ii) the $\operatorname{GGeo}(\tau, \sigma)$ distribution is an extremal member of the family of two parallel phase geometric distributions with the same mean and SCV, where one of the phase rates bursts ("mathematically") to +o with probability $(1-\tau)$;

iii) the underlying renewal process of a $\operatorname{GGeo}(\tau, \sigma)$ distribution has, as the counting process for the total number of events in $n$ consecutive slots, a compound binomial distribution with parameters $(n, \tau, \sigma)$.

For $C_{W}^{2}<|1-v|$, the GGeo distribution cannot be interpreted physically as a stochastic model. However, it can be considered meaningfully as pseudo-distribution function of a flow model approximation to an underlying stochastic model in which negative branching pseudoprobabilities (or weights) are permitted. In this sense, all analytical GGeo results, both exact and approximate, which are obtained for discrete time queueing systems and networks when $C_{W}^{2} \geq|1-v|$, may be used also as useful heuristic approximations when $C_{W}^{2}<|1-v|$. Note that the utility of other types of pseudo-distributions in systems modelling has been suggested by various authors (cf $[23,24])$. 
In the context of an ATM switch architecture, due to the fixed cell size and the nature of the associated output links, transmission times are assumed to be deterministic, with $\mathrm{SCV}=0$. It is interesting to note in this case that, if $\nu=1$, then $\tau=1$ and $\sigma=1$ and the $\operatorname{GGeo}(\tau, \sigma)$ distribution reduces to a true deterministic (D) distribution.

\section{ME Analysis of a Shared Buffer Queue: An Outline}

\subsection{Model Formulation and Notation}

Consider a general queueing model of a shared buffer switch with bursty arrivals and DF buffer management policy, depicted in Figure 3. The queueing model consists of $R$ parallel single server queues, where $R$ is the number of output ports. Each server represents an output port and each queue corresponds to the address queue for the output port. There are $R$ bursty and heterogeneous arrival streams of cells, one stream to each of $R$ input ports. Each stream is described by a GGeo $\left(\tau_{a i}, \sigma_{a i}\right)$ distribution with mean arrival rate $\Lambda_{i}$ cells per slot and SCV of interarrival time $\mathrm{C}_{a i}^{2}$ for stream $i, i=1,2, \ldots, R$. Similarly, the transmission (or service) time of a cell at queue $i$ follows a $\operatorname{GGeo}\left(\tau_{s i}, \sigma_{s i}\right)$ distribution with mean rate $\mu_{i}$ cells per slot and SCV of interdeparture time $\mathrm{C}_{s i}^{2}$ for stream $i, i=1,2, \ldots, R$. Let $N$ be the size of the total shared buffer. A cell is lost if it arrives at a time when there are a total of $N$ cells in the $R$ queues. Without loss of generality, it is assumed that any of the $R$ queues may attain the maximum size $N$.

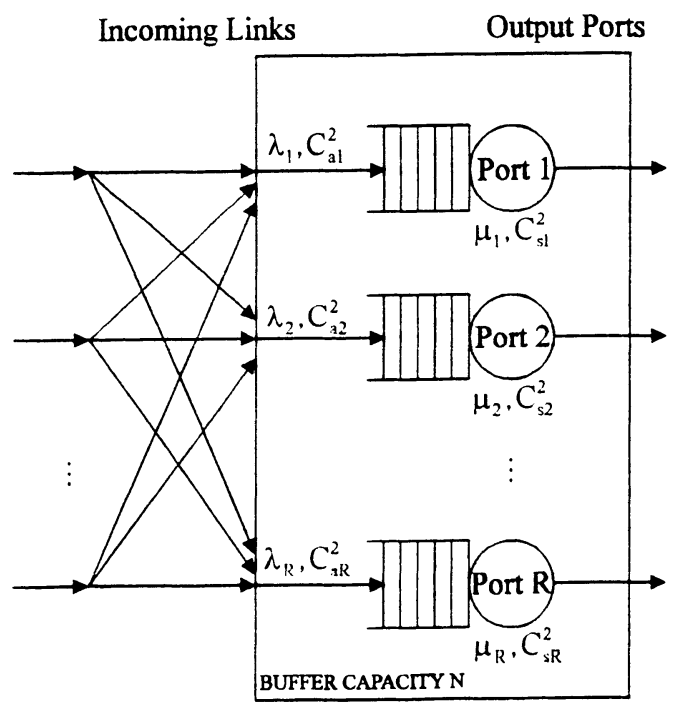

Figure 3. The $\mathrm{S}_{\mathrm{R} \times \mathrm{R}}(\mathrm{GGeo} / \mathrm{GGeo} / 1) / \mathrm{N}$ queueing model of a shared buffer switch.

The queueing model of the shared buffer switch is denoted by $S_{\mathrm{R} \times \mathrm{R}}(\mathrm{GGeo} / \mathrm{GGeo} / 1) / \mathrm{N}$, such that 
i) the overall (merged) interarrival times and service times at a $R \times R$ switch queue are heterogeneous and GGeo distributed,

ii) each output port has a single server,

iii) the total buffer capacity of the switch is $N$.

Finally, let the state of the system at any time be represented by a vector $\boldsymbol{n}=\left(n_{1}, n_{2}, \ldots, n_{R}\right)$, where $n_{i}$ is the number of cells in queue $i, i=1,2, \ldots, R$.

\subsection{A ME Solution for the $\mathrm{S}_{\mathrm{R} \times \mathrm{R}}(\mathrm{GGeo} / \mathrm{GGeo} / 1) / \mathrm{N}$ Queueing Model}

The form of the ME solution $p(\boldsymbol{n})$,

$\boldsymbol{n} \in \mathrm{S}(N, R)=\left\{\boldsymbol{n}=\left(n_{1}, n_{2}, \ldots, n_{R}\right): \sum_{i=1}^{R} n_{i} \leq N, 0 \leq n_{i} \leq N, i=1, \ldots, R\right\}$ of $\mathrm{S}_{\mathrm{R} \times \mathrm{R}}(\mathrm{GGeo} / \mathrm{GGeo} / 1) / \mathrm{N}$ queueing model has been characterised in [16] subject to normalisation and the constraints: server utilisation $U_{i}, 0<U_{i}<1$; mean queue length $L_{i}, U_{i} \leq L_{i}<N$; aggregate probability $\varphi_{i}$ of full buffer with $n_{i}>0,0<\varphi_{i}<1, i=1,2, \ldots, R$, and is given - by the method of Lagrange's undetermined multipliers - as

$$
\mathrm{p}(\boldsymbol{n})=\frac{1}{Z} \prod_{i=1}^{R} g_{i}^{\mathrm{s}_{i}(\boldsymbol{n})} x_{i}^{n_{i}} y_{i}^{\mathrm{f}_{i}(\boldsymbol{n})}, \quad \forall \boldsymbol{n} \in \mathrm{S}(N, R)
$$

where $Z$ is the normalising constant

$$
Z=\sum_{n \in \mathrm{S}(N, R)} \prod_{i=1}^{R} g_{i}^{\mathrm{s}_{i}(n)} x_{i}^{n_{i}} y_{i}^{\mathrm{f}_{i}(n)}
$$

and $\mathrm{s}_{i}(\mathbf{n})$ and $\mathrm{f}_{i}(\mathbf{n})$ are the auxiliary (indicator) functions

$$
\begin{aligned}
& \mathrm{s}_{i}(\boldsymbol{n})= \begin{cases}1 & n_{i}>0 \\
0 & \text { otherwise }\end{cases} \\
& \mathrm{f}_{i}(\boldsymbol{n})= \begin{cases}1 & \sum_{j=1}^{R} n_{j}=N \wedge \mathrm{s}_{i}(\boldsymbol{n})=1 \\
0 & \text { otherwise }\end{cases}
\end{aligned}
$$

and $\left\{g_{i}, x_{i}, y_{i}: i=1,2, \ldots, R\right\}$ are the GGeo type Lagrangian coefficients corresponding to the constraints $\left\{U_{i}, L_{i}, \varphi_{i}: i=1,2, \ldots, R\right\}$.

The GGeo Lagrangian coefficients $\left\{g_{i}, x_{i}: i=1,2, \ldots, R\right\}$ are obtained by making asymptotic connections with the GGeo/GGeo/1 queue [18], namely

$$
g_{i}=\frac{\rho_{i}\left(1-x_{i}\right)}{x_{i}\left(1-\rho_{i}\right)}, \quad x_{i}=\frac{\tilde{L}_{i}-\rho_{i}}{\tilde{L}_{i}}, \quad i=1,2, \ldots, R,
$$

where $\tilde{L}_{i}$ is the mean queue length of the GGeo/GGeo/1 queue and is given by

$$
\tilde{L}_{i}=\frac{\rho_{i}}{2}\left(1+\frac{C_{a i}^{2}+\rho_{i} C_{s i}^{2}}{1-\rho_{i}}\right) \text {, where } \rho_{i}=\Lambda_{i} / \mu_{i} \text {. }
$$


The GGeo type Lagrangian coefficients $\left\{y_{i}: i=1,2, \ldots, R\right\}$ can be computed by

i) focusing on the flow balance equations

$$
\Lambda_{i}\left(1-\pi_{i}\right)=\mu_{i} U_{i}, \quad i=1,2, \ldots, R
$$

where $\pi_{i}$ is the cell loss probability for attempted arrivals to the queue for output port $i$,

ii) deriving recursive expressions for $\pi_{i}$ and $U_{i}, 1,2, \ldots, R$, and

iii) solving numerically the resultant non-linear simultaneous equations.

The normalising constant can be determined by applying the generating function approach [25] and can be computed recursively as shown below.

$Z=\sum_{v=0}^{N-1} C_{1}(v)+C_{2}(N)$

where $\left\{C_{1}(v): v=0,1, \ldots, N-1\right\}$ and $C_{2}(N)$ are determined via the following recursive formulae.

$C_{1}(\mathrm{v})=C_{1 R}(\mathrm{v}), \quad \mathrm{v}=0,1 \ldots, N-1$

$C_{2}(N)=C_{2 R}(N)$

where

$C_{k r}(v)=C_{k, r-1}(v)-\left(1-B_{r}\right) x_{r} C_{k, r-1}(v-1)+x_{r} C_{k r}(v-1)$,

$B_{r}= \begin{cases}g_{r} & k=1 \\ g_{r} y_{r} & k=2\end{cases}$

for $k=1,2, r=2, \ldots, R, v=1,2, \ldots, N-2+k$, with initial conditions

$C_{k 1}(v)= \begin{cases}1 & v=0 \\ B_{1} x_{1}^{v} & v=1,2, \ldots, N-2+k\end{cases}$

$C_{k r}(0)=1, \quad k=1,2, \quad r=2, \ldots, R$.

Similarly the utilisation $U_{i}$ can be expressed as

$U_{i}=\frac{1}{Z}\left(\sum_{v=1}^{N-1} C_{1}^{(i)}(v)+C_{2}^{(i)}(N)\right), \quad i=1,2, \ldots, R$

where

$C_{k}^{(i)}(v)=\left(1-B_{i}\right) x_{i} C_{k}^{(i)}(v-1)+B_{i} x_{i} C_{k}(v-1), \quad v=2, \ldots, N-2+k, \quad k=1,2, \quad i=1, \ldots, R$,

with initial conditions $C_{k}^{(i)}(1)=B_{i} x_{i}$.

The marginal state probabilities can be determined by using ME solution (4.1) and the recursive expressions (above) for $C_{k}^{(i)}(v)$. Let $n(i)$ be the random variable for the number of cells at queue $i, i=1,2, \ldots, R$. Then the marginal state probabilities are given by [16]

$\operatorname{Pr}\left[n(i)=l_{i}\right]=\operatorname{Pr}\left[n(i) \geq l_{i}\right]-\operatorname{Pr}\left[n(i) \geq l_{i}+1\right]$

where 
$\operatorname{Pr}\left[n(i) \geq l_{i}\right]=\frac{x_{i}^{l_{i}-1}}{Z}\left(\sum_{v=l_{i}}^{N-1} C_{1}^{(i)}\left(v-l_{i}+1\right)+C_{2}^{(i)}\left(N-l_{i}+1\right)\right)$

$i=1,2, \ldots, R, l_{i}=1,2, \ldots, N$.

Moreover, by focusing on a stable $\mathrm{S}_{\mathrm{R} \times \mathrm{R}}(\mathrm{GGeo} / \mathrm{GGeo} / 1) / \mathrm{N}$ queue under DF policy and by using GGeo type probabilistic arguments, the marginal cell-loss probabilities are seen to be [16]

$\pi_{i}=\frac{1}{Z}\left(F_{i}(N)+C_{2}(N)-\mu_{i} \tau_{a i} \delta_{i} C_{2}^{(i)}(N)\right)$

where

$\delta_{i}=\frac{\tau_{s i}}{\tau_{s i}\left(1-\tau_{a i}\right)+\tau_{a i}}$

and

$F_{i}(N)=\delta_{i} \sum_{v=0}^{N-1} C_{1}(v)\left(1-\tau_{a i}\right)^{N-v}+\left(\left(1-\delta_{i}\right)\left(1-\sigma_{s i}\right)-\sigma_{s i} \delta_{i} \tau_{a i}\right) \sum_{v=1}^{N-1} C_{1}^{(i)}(v)\left(1-\tau_{a i}\right)^{N-v}, i=1,2, \ldots, R$.

The GGeo type Lagrangian coefficients $y_{i}$ can be determined numerically by substituting $U_{i}$ of (4.5) and $\pi_{i}$ of (4.7) in the flow balance conditions (4.3) and solving the resulting system of $R$ non-linear equations with $R$ unknowns $\left\{y_{i}: i=1,2, \ldots, R\right\}$, namely

$\left(1-\mu_{i} \tau_{a i} \delta_{i} \rho_{i}\right) C_{2}^{(i)}(N)=\rho_{i}\left(\sum_{v=1}^{N-1} C_{1}(v)-F_{i}(N)\right)-\sum_{v=1}^{N-1} C_{1}^{(i)}(v)$

for all $i=1,2, \ldots, R$ and $N \geq 2$.

System (4.8) can be solved by applying the numerical algorithm of Newton-Raphson, which is generally expected to give quadratic convergence. One significant limitation of this method is the requirement that the partial derivatives of the Jacobian matrix must be calculated at each iteration. However, this requirement may be avoided by applying an efficient recursive scheme (cf [15]). Thus, because of the recursive nature of the z-transforms which are used in the computational implementation of the ME solution, the $\mathrm{S}_{\mathrm{R} \times \mathrm{R}}(\mathrm{GGeo} / \mathrm{GGeo} / 1) / \mathrm{N}$ queueing model can be used as an effective building block in the analysis of large discrete time queueing networks of shared buffer ATM switches. The construction of such computational procedure is the subject of the following section.

\section{Arbitrary Open Queueing Networks of GGeo Type Shared Buffer Switches}

Consider an arbitrary discrete time open queueing network at equilibrium consisting of $M$ nodes, as depicted in Figure 4 . Each node $i, i=1,2, \ldots, M$, is a $R_{i} \times R_{i}$ shared buffer queueing model with finite capacity $N_{i}$ (see Figure 3 ). At any given time, the joint state of the network is denoted by $\boldsymbol{n}=\left(\boldsymbol{n}_{1}, \boldsymbol{n}_{2}, \ldots, \boldsymbol{n}_{M}\right)$ where $\boldsymbol{n}_{i}=\left(n_{i 1}, n_{i 2}, \ldots, n_{i R_{i}}\right)$ is the joint state of shared buffer queueing system $i$ and $n_{i j}$ is the number of cells queueing for output port $j, j=1,2, \ldots, R_{i}$. Moreover, let $\mathrm{p}(\boldsymbol{n})$ be the joint state probability of the network. 


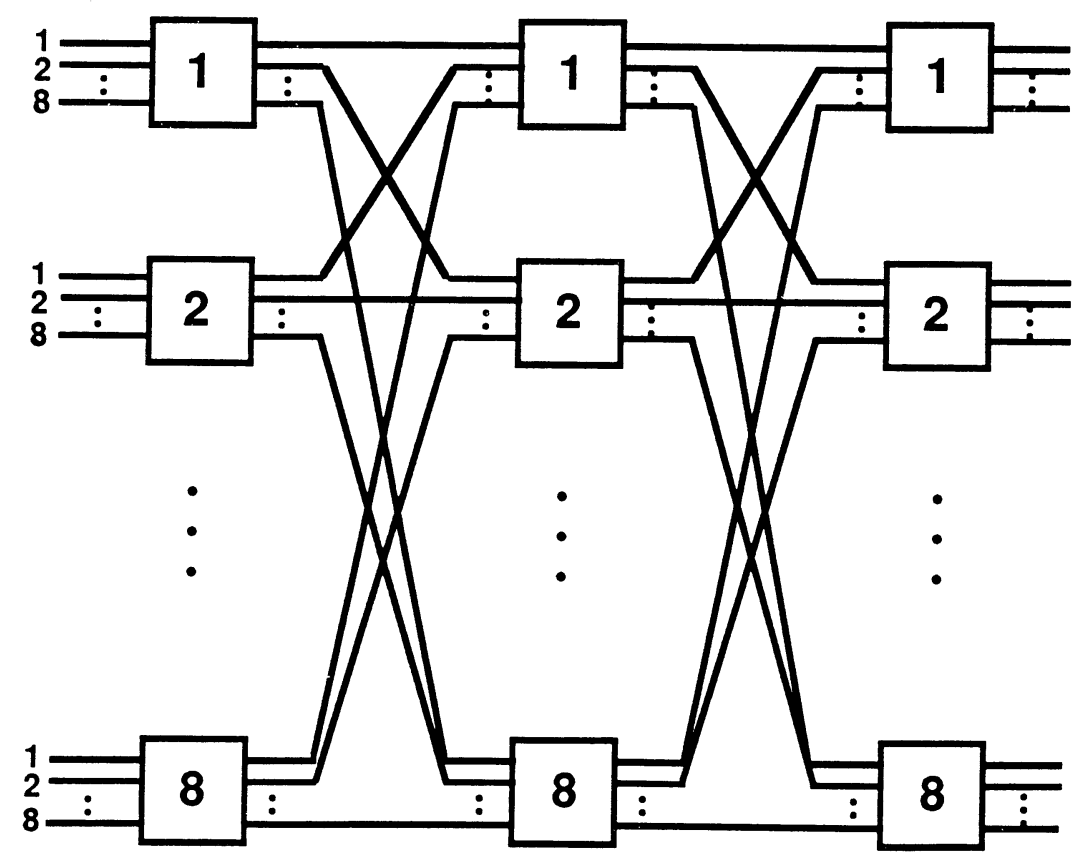

Figure 4. A network configuration of shared buffer switches.

The form of the ME solution, $\mathrm{p}(\boldsymbol{n})$, for a FCFS GGeo type open queueing network, subject to normalisation and the marginal constraints of shared buffer queueing systems used in Section 4, namely utilisation, $U_{i j}$, $0<U_{i j}<1$, mean queue length, $L_{i j}, U_{i j}<L_{i j}<N_{i}$, and aggregate full buffer probability with $n_{i j}>0, \varphi_{i j}, 0<\varphi_{i j}<1, j=1,2, \ldots, R_{i}, i=1,2, \ldots, M$, is given - by the method of Lagrange's undetermined multipliers — as

$\mathrm{p}(\boldsymbol{n})=\frac{1}{Z} \prod_{i=1}^{M} \prod_{j=1}^{R_{i}} g_{i j}^{\mathrm{s}_{i j}\left(\boldsymbol{n}_{i}\right)} x_{i j}^{n_{i j}} y_{i j}^{\mathrm{f}_{i j}\left(\boldsymbol{n}_{i}\right)}$

where $Z$ is the normalising constant and $\left\{g_{i j}, x_{i j}, y_{i j}\right\}$ are the Lagrangian coefficients corresponding to constraints $\left\{U_{i j}, L_{i j}, \varphi_{i j}\right\}$ respectively. The form of ME solution (5.1) clearly suggests a product form approximation, namely

$\mathrm{p}(\boldsymbol{n})=\prod_{i=1}^{M} \mathrm{p}\left(\boldsymbol{n}_{i}\right)$

where $\mathrm{p}\left(\boldsymbol{n}_{\boldsymbol{i}}\right)$ is determined by the ME solution (4.1) of each shared buffer $\mathrm{S}_{\mathrm{R} \times \mathrm{R}}(\mathrm{GGeo} / \mathrm{GGeo} / 1) / N_{i}$ queueing model, $i=1,2, \ldots, M$. 
The ME solution (5.1) can be implemented computationally by decomposing the network into individual building blocks of $\mathrm{S}_{R_{i} \times R_{i}}(\mathrm{GGeo} / \mathrm{GGeo} / 1) / N_{i}$ queues, $i=1,2, \ldots, M$, each of which can be solved in isolation by determining iteratively the first two moments of the overall flows in the network. Appropriate GGeo type flow formulae have been derived in [18], whilst Generalised Exponential (GE) type flow formulae and numerical approximations can be seen in [11] and [26], respectively.

In the sequel, routing of cells through the network is based upon the notion of the virtual circuit (VC). A VC has a fixed path through the network. All cells that belong to a particular VC flow along its path. A number of different VCs will exist across the network. It is assumed that the first two moments of the external flow of each VC as it arrives at the network is known. To this end, GGeo type ME solution and GGeo flow formulae can be applied in a similar fashion to that established in [17]. However, the flow of cells belonging to VCs must be converted to flows through each switch/port, and from one switch/port to another. Due to finite buffer sizes cell loss will occur at switches and thus within a VC the flow of cells will reduce at each link composing its path. Because cell flows are attenuated, it is not possible to calculate apriori the flows required in the GGeo flow formulae.

The path a VC can be represented as an ordered and finite list of switch/port pairs,

$(0,0) \rightarrow(i, a) \rightarrow(j, b) \rightarrow(k, c) \rightarrow, .,(n, f) \rightarrow(0,0)$

where $(0,0)$ represents the outside world. Figure 5 shows two VCs, namely $(0,0) \rightarrow(1,3) \rightarrow(2,1) \rightarrow(0,0), \quad$ and $\quad(0,0) \rightarrow(1,1) \rightarrow(2,2) \rightarrow(3,2) \rightarrow(0,0) \quad$ following predefined routes through three $3 \times 3$ switches.

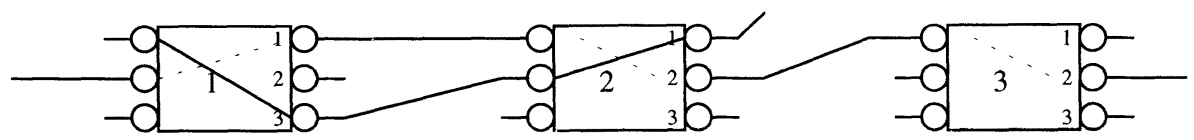

Figure 5. Routes of VCs across a Network of ATM Switches.

The mean rate of cells flowing on a link, say $(\mathrm{j}, \mathrm{b}),(\mathrm{k}, \mathrm{c})$, of the path of $\mathrm{VCl}$ is given by

$$
\Lambda_{(j, b),(k, c)}^{\mathrm{vc} \ell}=\left(1-\pi_{(i, a),(j, b)}\right) \Lambda_{(i, a),(j, b)}^{\mathrm{vc \ell}}
$$

where $\Lambda_{(i, a),(j, b)}^{\mathrm{vc}}$ is the mean overall arrival rate of cells on the link $(i, a),(j, b)$ of $\mathrm{VCl}$ and $\pi_{(i, a),(j, b)}$ is the cell loss (or blocking) probability that cells from switch/port (i,a) are lost on arrival to switch/port (j,b). It can be shown, by using GGeo type probabilistic arguments (as in the case of a network with ordinary finite capacity queues [17]), that probability $\pi_{(i, a),(j, b)}$ can be obtained by equation (4.7) with the subscripts of parameters changing to $(i, a),(j, b)$ to reflect that arrivals to switch/port $(\mathrm{j}, \mathrm{b})$ are considered from one stream emanating from switch/port (i,a) instead of the merged stream of all arrivals. Once all the blocking 
probabilities are known the flow on each link of each VC can be calculated (starting with $\Lambda_{(0,0),(i, a)}^{\mathrm{vc} \ell}$, the known external arrival rate of $\mathrm{VCl}$ ) by repeated application of equation (5.3).

The mean flow of cells generated by switch/port (i,a) upon $\mathrm{VCl}$ and entering switch/port $(j, b)$ is referred to as the mean effective rate $\lambda_{(i, a),(j, b)}^{\mathrm{vc} \ell}$. This is the same as the mean rate of cells of $\mathrm{VCl}$ that leave switch/port $(\mathrm{j}, \mathrm{b})$. As this is the overall mean rate on the next link $(\mathrm{k}, \mathrm{c})$ of the path of $\mathrm{VCl}$, it follows that

$\lambda_{(i, a),(j, b)}^{\mathrm{vc} \ell}=\Lambda_{(j, b),(k, c)}^{\mathrm{vc} \ell}$

If $\mathrm{VCl}$ enters the network at switch/port (i,a), its mean overall external arrival rate is denoted by $\Lambda_{(0,0),(i, a)}^{\mathrm{vc} \ell}$, and its SCV by $C_{(0,0),(i, a)}^{\mathrm{vc} \ell}$. The mean effective arrival rate at switch/port $(\mathrm{i}, \mathrm{a})$, obtained via equation (5.3), is $\Lambda_{(i, a),(j, b)}^{\mathrm{vc},}$. By using the GGeo split flow formulae [18], the SCV of the effective external interarrival time at switch/port (i,a) is clearly given by

$\tilde{\mathrm{C}}_{(0,0),(i, a)}^{v c l}=\pi_{(0,0),(i, a)}+\mathrm{C}_{(0,0),(i, a)}^{v c l}\left(1-\pi_{(0,0),(i, a)}\right)$

The overall mean rate of cells that flow between upstream switch/port $(r, s)$ and switch/port $(\mathrm{k}, \mathrm{j})$ is given by

$\Lambda_{(r, s),(k, j)}=\sum_{l \in S_{(r, s)(k, j)}} \Lambda_{(r, s),(k, j)}^{\mathrm{v} l l}$

where $_{(r, s)(k, j)}$ is the set of all VCs $\{l\}$ having $(\mathrm{r}, \mathrm{s}),(\mathrm{k}, \mathrm{j})$ as a link on their path. Moreover, the overall mean arrival rate of cells at switch/port $(k, j)$ is given by

$\Lambda_{(k, j)}=\sum_{(r, s) \in S_{(k, j)}} \Lambda_{(r, s),(k, j)}$

where $s_{(k, j)}$ is the set of all upstream switch/ports $\{(\mathrm{r}, \mathrm{s})\}$ linking to switch/port $(\mathrm{k}, \mathrm{j})$.

To simplify notation, in what follows the suffix $(k, j)$ for port $j$ of switch $k$ is represented by $i$ and the suffix $(r, s)$ for port $s$ of switch $r$ is represented by $j$.

The SCV of effective interarrival times at output port $i$ is given by the merging of effective GGeo type streams [18]

$\tilde{\mathrm{C}}_{a i}^{2}=-1-\lambda_{i}+\left(\sum_{l \in S_{l i}^{a}} \frac{\lambda_{i i}^{v c l}\left(\tilde{\mathrm{C}}_{0 i}^{v c l}+\lambda_{0 i}^{v c l}+1\right)}{+}+\sum_{j \in A(i)} \frac{\lambda_{j i}}{\lambda_{i}\left(\tilde{\mathrm{C}} a_{j i}^{2}+\lambda_{j i}+1\right)}\right)^{-1}, i=1,2 \ldots, M$

where $s_{i t}^{a}$ is the set of VCs that enter the network at port i, $A(i)$ is the set of all upstream ports immediately connected to port $i, \lambda_{0 \mathrm{i}}^{\mathrm{vcl}}$ and $\tilde{\mathrm{C}}_{0 i}^{v c l}$ are the effective mean rate and SCV, respectively, of the interarrival times of cells entering the network at port $i$ upon $\mathrm{VCl}$, (cf, (5.4), (5.5)) and $\tilde{C} a_{j i}^{2}$ is the SCV of the effective stream of cells that leave port $j$ and enter port $i$. Clearly, 
$\lambda_{j i}=\left(1-\pi_{j i}\right) \Lambda_{j i}, j=0,1, . ., M, \quad i=1,2, . ., M$

where $\pi_{j i}$ is the blocking probability determined by (4.7) with parameters $\Lambda_{j i}$ and $\mathrm{C}_{d j i}^{2}$ the latter being the SCV of the interdeparture time of the overall stream of cells from port $j$ to port $i$.

The SCV of the overall interarrival times at output port $i$ is clearly given by the merging of overall GGeo type streams [18], namely

$\mathrm{C}_{a i}^{2}=-1-\Lambda_{i}+\left(\sum_{l \in S_{i i}^{a}} \frac{\Lambda_{0 i}^{v c l}}{\Lambda_{i}\left(C_{0 i}^{v c l}+\Lambda_{0 i}^{v c l}+1\right)}+\sum_{j \in A(i)} \frac{\Lambda_{j i}}{\Lambda_{i}\left(\mathrm{C}_{d j i}^{2}+\Lambda_{j i}+1\right)}\right)^{-1}, j=0,1, . ., M ; i=1,2, . ., M(5.10)$

The departure rate from (or the effective arrival rate to) an output port $j$ is a non-linear function of the traffic characteristics of the switching node and is given by the sum of the rates from that port to all downstream destinations

$\lambda_{j}=\sum_{l \in S_{i j}^{a}} \Lambda_{j 0}^{v l}+\sum_{i \in D(j)} \Lambda_{j i}, j=1,2, . ., M ; i=1,2, . ., M$

where $s_{i j}^{a}$ is the set of VCs that leave the network at port $\mathrm{j}$ and $\Lambda_{j 0}^{\mathrm{vc1}}$ is the rate at which cells leave the network from port $j$ upon $\mathrm{VCl}$ and $D(j)$ is the set of all downstream ports immediately connected to port $j$. Moreover, the SCV of the interdeparture time $\mathrm{C}_{d i}^{2}$ can be approximated by analysing the departure process of a stable GGeo/GGeo/1 queue with infinite capacity and effective revised arrival rate, $\lambda_{i}$ and is given by [18]

$\mathrm{C}_{d i}^{2}=\tilde{\rho}_{i}^{2}\left(\mathrm{C}_{s i}^{2}+\mu_{i}-1\right)-\tilde{\rho}_{i}\left(\tilde{\mathrm{C}}_{a i}^{2}+\lambda_{i}-1\right)+\tilde{\mathrm{C}}_{a i}^{2}, i=1,2, \ldots . M$

where $\tilde{\rho}_{i}=\frac{\lambda_{i}}{\mu_{i}}$.

The splitting of the interdeparture times at each output port $\mathrm{j}$ with parameters $\left(\Lambda_{j i}, \mathrm{C}_{d j i}^{2}\right)$ can be made dynamically via computational iteration which necessitates the estimation of transition probabilities $\alpha_{j i}$ that a departing cell from port $\mathrm{j}$ will go to port i. Probabilities $\left\{\alpha_{j i}\right\}$ can be determined by observing that each output port i experiences a virtual arrival process of cells complying with a random routing policy and is clearly given by

$\alpha_{j i}=\frac{\Lambda_{j i}}{\lambda_{j}}$

The SCV of interdeparture time for the overall flow is given by

$\mathrm{C}_{d j i}^{2}=1+\alpha_{j i}\left(\mathrm{C}_{d j}^{2}-1\right), j=1,2, . ., M ; i=0,1, . ., M$

whilst, the SCV of effective interarrival time at port $i$ from port $j$ can be expressed by

$\tilde{\mathrm{C}}_{a j i}^{2}=\pi_{j i}+\mathrm{C}_{d j i}^{2}\left(1-\pi_{j i}\right), j=1,2, . ., M ; i=1,2, . ., M$ 


\section{A ME Algorithm for the Analysis of an Arbitrary Open Network of Shared Buffer GGeo Type Queues}

This section presents an algorithm for the solution of a FCFS open network of shared buffer switches. The building blocks of the ME algorithm are based upon

i) the ME solution of the $S_{R \times R}(G G e o / G G e o / 1) / N$ shared buffer queuing model,

ii) the GGeo type flow approximation formulae for calculation of the mean and SCV of the interarrival and interdeparture times at each output port and

iii) the mean flow rates of VCs on each link of their paths .

The steps of the algorithm, which broadly follow those established by the ME methodology as applied to simpler types of networks of ordinary finite queues [14,17], are presented below.

Step 0. Read in input parameters and routes of VCs and service time parameters of output port queues.

Step 1. Initialise all cell loss probabilities to zero. Set SCV of interdeparture times to 1.

Step 2. Compute the mean flows rates for each VC on each link in their paths using equation (5.3) and calculate effective flows of each VC as it enters the network using equations (5.3) and (5.5).

Step 3. Calculate the overall and effective mean arrival rate to each output port from all other output ports and the overall mean departure rate from each output port to all other output ports using equations (5.4), (5.6), (5.7), (5.9) and (5.11).

Step 4. Obtain the routing probabilities via equation (5.13).

Step 5. Utilise the routing probabilities to obtain the SCV of interdeparture times from each output port to other output ports via equations (5.14).

Step 6. Calculate the SCV of the merging streams (overall and effective) at each output port using equations (5.8), (5.10) and(5.15).

Step 7. Obtain the queue length distribution of the $\mathrm{S}_{\mathrm{R} \times \mathrm{R}}(\mathrm{GGeo} / \mathrm{GGeo} / 1) / \mathrm{N}$ as described in Section 4 (cf equations $(4.1,4.8)$ ) with the overall interarrival time parameters obtained in steps 3 and 6.

Step 8. Compute the blocking probabilities using equation (4.7).

Step 9. Estimate the overall SCV of the interdeparture time of each output port using equation (5.12).

Repeat steps 2-9 until convergence of the calculated value of the SCV of the interdeparture time.

Step 10. Obtain cell loss probability and mean delay for each output port in the network.

\section{$\underline{\text { Remarks }}$}

The main computational cost of the ME algorithm is the calculation of cell loss probabilities at the output ports of the shared buffer switch, which must be obtained at each iteration (step 8). Kouvatsos et al [16] have shown experimentally, using a Sun work station, that the computational cost for solving a $S_{\mathrm{R} \times \mathrm{R}}(\mathrm{GGeo} / \mathrm{GGeo} / 1) / \mathrm{N}$ queue 
remains extremely small due to the recursive nature of the z-transforms used in implementation of the ME solution. For example, for $R=50$ and $N=100$ the cost just approaches 37 seconds of CPU time! Thus, using the $S_{R \times R}(G G e o / G G e o / 1) / N$ queue as an effective building block, the performance analysis of quite complex networks of shared buffer queues can be performed in a few minutes.

The existence and uniqueness of the solution of the system of non-linear equations (4.8) cannot be proved analytically due to the complexity of the expressions involved. Moreover, no rigorous mathematical justification can be given for convergence of the $\mathrm{C}_{d i}{ }^{2}$ 's. Nevertheless, there has been no incidence of numerical instability and no lack of convergence in the many experiments which have been performed.

\section{Numerical Results}

In this section, typical numerical results are included in order to demonstrate the utility and computational efficiency of the ME algorithm, as applied to arbitrary open networks of shared buffer queues in the discrete time domain. Notably, the credibility of the building blocks, namely

i) the ME solution of each $S_{\mathrm{R} \times \mathrm{R}}(\mathrm{GGeo} / \mathrm{GGeo} / 1) / \mathrm{N}$ queue in isolation and

ii) the GGeo type flow formulae for the first two moments of the interdeparture, split and merge processes within an arbitrary FCFS open network of ordinary single server finite capacity GGeo type queues under DF policy

have been experimentally verified earlier in [16] and [17], respectively, against simulation at 95\% confidence intervals by making use of the Queueing Network Analysis Package (QNAP-2) [27].

The ME algorithm for arbitrary FCFS open discrete time queueing networks of shared buffer ATM switches under DF policy was implemented on a Sun workstation. The bursty nature of the interarrival time at each output port queue is approximated by a GGeo distribution. The constant transmission (service) time distributions are represented by GGeo pseudo-distributions with $\mathrm{C}_{s i}^{2}=0$ and $\mu_{i}=1$.

The utility of the ME algorithm is shown in Tables 1-2, representing a queueing network of three shared buffer switches with input data and output performance metrics in terms of cell loss and mean delay. It is assumed that only one link exists between one switch and the next. The ME algorithm has also been employed to investigate the behaviour of a single shared buffer switch within various open GGeo type queueing networks with arbitrary configurations. It has been verified experimentally that, as in the case of a single independent shared buffer switch [16], the ME results exhibit the following features:

i) for "small" cell loss probaibilities (e.g. $10^{-1}, 10^{-2}, \ldots$ ), the relationship between cell loss probability and buffer capacity is log-linear, as expected;

ii) for a target cell loss probability (say $10^{-6}$ ), the relationships between interarrival time SCVs and

a) optimal buffer capacity and

b) mean delay

are linear. 
Table 1. Service parameters and output statistics of the three $3 \times 3$ shared buffer switches

\begin{tabular}{|c|c|c|c|c|c|}
\hline \multicolumn{5}{|c|}{ input data } & \multicolumn{2}{c|}{ output statistics } \\
\hline switch & port & $\mu$ & $\mathrm{C}_{s}^{2}$ & cell loss prob. & mean delay \\
\hline \multirow{3}{*}{1} & 1 & 1.0 & 0 & 0.119 & 3.195 \\
\cline { 2 - 6 } & 2 & 1.0 & 0 & 0.018 & 1.635 \\
\cline { 2 - 6 } & 3 & 1.0 & 0 & 0.097 & 3.468 \\
\hline \multirow{3}{*}{2} & 1 & 1.0 & 0 & 0.295 & 4.076 \\
\cline { 2 - 6 } & 2 & 1.0 & 0 & 0.037 & 1.891 \\
\cline { 2 - 6 } & 3 & 1.0 & 0 & 0.329 & 4.026 \\
\hline \multirow{3}{*}{3} & 1 & 1.0 & 0 & 0.215 & 3.447 \\
\cline { 2 - 6 } & 2 & 1.0 & 0 & 0.030 & 2.601 \\
\cline { 2 - 6 } & 3 & 1.0 & 0 & 0.304 & 3.575 \\
\hline
\end{tabular}

Table 2. VC external traffic characteristics (mean rate $\Lambda_{0}, \mathrm{SCV} \mathrm{C}_{0}^{2}$ ) and paths across the network. (Key $(i, j)$ : (switch $i$, ouput port $j$ ).

\begin{tabular}{|c|c|c|cccc|}
\hline $\mathrm{VC}$ & $\Lambda_{0}$ & $\mathrm{C}_{0}^{2}$ & \multicolumn{4}{|c|}{ Path of VC } \\
\hline 1 & 0.2 & 10 & $(1,2)$ & & \\
\hline 2 & 0.3 & 10 & $(2,2)$ & & \\
\hline 3 & 0.4 & 10 & $(3,2)$ & & \\
\hline 4 & 0.2 & 10 & $(2,1)$ & $\rightarrow(3,2)$ & \\
\hline 5 & 0.12 & 20 & $(1,3)$ & $\rightarrow(3,3)$ & $\rightarrow(2,2)$ \\
\hline 6 & 0.13 & 30 & $(2,1)$ & $\rightarrow(3,1)$ & $\rightarrow(1,2)$ \\
\hline 7 & 0.14 & 40 & $(2,3)$ & $\rightarrow(1,3)$ & $\rightarrow(2,2)$ \\
\hline 8 & 0.3 & 50 & $(3,1)$ & $\rightarrow(1,1)$ & $\rightarrow(2,2)$ \\
\hline 9 & 0.16 & 60 & $(3,3)$ & $\rightarrow(2,3)$ & $\rightarrow(1,2)$ \\
\hline 10 & 0.17 & 15 & $(1,1)$ & $\rightarrow(2,2)$ & \\
\hline 11 & 0.18 & 20 & $(1,3)$ & $\rightarrow(3,2)$ & \\
\hline 12 & 0.09 & 10 & $(2,1)$ & $\rightarrow(3,2)$ & \\
\hline 13 & 0.2 & 15 & $(2,3)$ & $\rightarrow(1,2)$ & \\
\hline 14 & 0.01 & 100 & $(3,1)$ & $\rightarrow(1,2)$ & \\
\hline 15 & 0.11 & 130 & $(3,3) \rightarrow(2,2)$ & \\
\hline
\end{tabular}


Thus, it is implied that the optimal buffer capacity and mean delay within a network can be determined easily for even smaller cell loss probabilities for any given SCVs of interarrival times.

Finally, Figure 6 shows the low computational cost of implementing the ME algorithm against the number of shared buffer switches in an arbitrary FCFS open network.

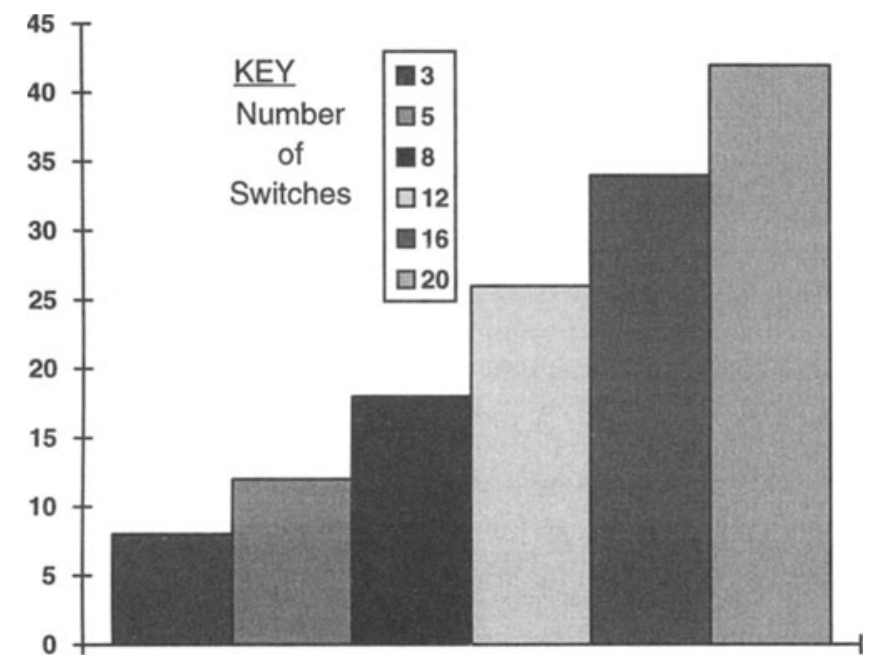

Figure 3. CPU time (seconds) of ME algorithm against number of $8 \times 8$ shared buffer switches of arbitrary QNMs.

\section{Conclusions}

A ME product form approximation is proposed for arbitrary FCFS open discrete time QNMs of GGeo shared buffer queues. Analytic ME solutions for a shared buffer $\mathrm{S}_{\mathrm{R} \times \mathrm{R}}(\mathrm{GGeo} / \mathrm{GGeo} / 1) / \mathrm{N}$ queueing model in conjunction with GGeo type formulae for the first two moments of the discrete time flows at each queue play the rôle of building blocks in the decomposition process of the network. The low computational cost of the ME algorithm makes it practical to analyse arbitrarily complex ATM networks of shared buffer switches.

The ME algorithm can be extended to analyse shared buffer ATM switch architectures under different buffer management simultaneity policies and multiple class streams of arriving cells at each output (or input) port. Entropy maximisation can also be applied to analyse ATM switches with push-out space priorities. Moreover, closed form performance expressions for discrete time queueing models of ATM switches with bursty and correlated traffic can be derived based on batch renewal processes and the GGeo bulk time distribution. Extensions of this kind are the subject of current study. 


\section{References}

[1] F.A. Tobagi, "Fast packet switch architecture's for broadband integrated services networks", Proc. IEEE 78: pp.1133-1167, January 1990.

[2] M. Devault, J.-Y. Cochennec and M. Servel, "The Prelude ATD experiment: Assessments and future prospects”, IEEE J. SAC 6(9), pp.1528-1537, December 1988.

[3] H. Kuwaha, N. Endo, M. Ogino and T. Kozaki, "A Shared Buffer Memory Switch for an ATM Exchange", Int. Conf. on Communications, pp.441-445, Boston, MA, June 1989

[4] H. Lee, K. Kook, C.S. Rim, K. Jun and S-K. Lim, "A Limited Shared Output Buffer Switch for ATM", Fourth In. Conf. on Data Communication Systems and their Performance, pp.163-179, Barcelona, 1990

[5] A.E. Eckberg and T-C. Hou, "Effects of Output Buffer Sharing on Buffer Requirements in an ATDM Packet Switch", INFOCOM '88, pp.459-466, March 1988

[6] G.H. Petit and E.M. Desmet, "Performance Evaluation of Shared Buffer Multiserver Output Queue Switches used in ATM", 7th. ITC Specialist Seminar, paper 7.1, New Jersey, 1990

[7] P.G. Harrison and A. de C. Pinto, "Blocking in Asynchronous Buffered Banyan Networks", Proc. Int. Seminar, Kyoto, Japan, December 1991

[8] H. Yamashita, H.G. Perros, S-W. Hong\}, "Performance Modelling of a Shared Buffer ATM Switch Architecture", ITC-13, Teletraffic and Datatraffic in a Period of Change, ed. Jensen and Iverson, North-Holland, pp.993-999, 1991

[9] S-W. Hong, H.G. Perros, H. Yamashita, "A Discrete-Time Queueing Model of the Shared Buffer ATM Switch with Bursty Arrivals”', Research Report, Computer Science Dept., North Carolina State University, 1992

[10] E.T. Jaynes, "Information Theory and Statistical Mechanics", Phys. Rev. 106(4), pp.620-630, 1957

[11] D.D. Kouvatsos, "Maximum Entropy Methods for General Queueing Networks", in Modelling Techniques and Tools for Performance Analysis, ed. D. Potier, NorthHolland, pp.589-609, 1985

[12] D.D. Kouvatsos, "Maximum Entropy and the G/G/1/N Queue", Acta Informatica 23, pp.545-565, 1986

[13] D.D. Kouvatsos, "A Maximum Entropy Analysis of the G/G/1 Queue at Equilibrium", J. Op. Res. Soc. 39(2), pp.183-200, 1989

[14] D. D. Kouvatsos and N.P. Xenios, "MEM for Arbitrary Queueing Networks with Multiple General Servers and Repetitive Service Blocking”, Perf. Eval. 10, pp.169-195, 1989

[15] D.D. Kouvatsos and S.G. Denazis, "A Universal Building Block for the Approximate Analysis of a Shared Buffer ATM Switch Architecture", Annals of Operations Research 44, Special Issue on Performance Analysis of High Speed Networks, pp.241-278, 1994

[16] D. D. Kouvatsos, N. M. Tabel-Aouel and S.G. Denazis, "ME Based Approximations for General Discrete-Time Queueing Models”, Perf. Eval. 21, pp.81-109, 1994 
[17] D.D. Kouvatsos, N. M. Tabel-Aouel and S.G. Denazis, "Approximate Analysis of Discrete-Time Queueing Networks with or without Blocking”, IFIP Transactions C-21, Special Issue on High Speed Networks and their Performance, North-Holland, pp.399-434, 1994

[18] D.D. Kouvatsos and N.M. Tabet-Aouel, "GGeo-type Approximations for General Discrete-Time Queuing Systems”, IFIP Transactions C-15, Special Issue on Modelling and Performance Evaluation of ATM Technology, North-Holland, pp.469-483, 1993

[19] A. Gravey, G. Hebuterne, “'Simultaneity in Discrete Time Single Server Queues with Bernoulli Inputs”', Performance Evaluation 14, pp.123-131, 1992

[20] D.D. Kouvatsos, "Cost-Effective Solutions for Queueing Models of ATM Networks", in Tutorial Proceedings of 2nd IFIP Workshop on the Performance Modelling and Evaluation of ATM Networks, ed. D.D. Kouvatsos, University of Bradford, pp.8/1-8/40, 4-7th July, 1994.

[21] E.T. Jaynes, "Information Theory and Statistical Mechanics II", Phys. Rev. 108, pp.171-190, 1957

[22] J.E. Shore and R.W. Johnson, "Axiomatic Derivation of the Principle of Maximum Entropy and the Principle of Minimum Cross-Entropy", IEEE Trans. Inf. Theory IT-26, pp.26-27, 1980

[23] C. Saur, "Configuration of Computing Systems: An Approach Using Queueing Network Models”, Ph.D. Thesis, University of Texas, 1975

[24] S. Nojo and H. Watanabe, "A New Stage Method of getting Arbitrary Coefficient of Variation by Two Stages”, Trans. IEICE 70, pp.33-36, 1987

[25] A.C. Williams and R.A. Bhandiwad, "A Generating Function Approach to Queueing Network Analysis of Multiprogrammed Computers", Networks 6, pp.1-22, January 1976

[26] Afonso de Compos Pinto "Models of Finite-buffered Packet-switched Multistaged Interconnection Networks" PhD Thesis, University of London, Imperial College of Science, Technical and Medicine (Dept. of Computing), 1994

[27] M. Veran and D. Potier, "QNAP-2: A Portable Environment for Queueing Network Modelling", in Modelling Techniques and Tools for Performance Analysis, ed. D. Potier, North-Holland, pp.25-63, 1985 